\title{
Rest and treatment/rehabilitation following sport-related concussion: a systematic review
}

\author{
Kathryn J Schneider, ${ }_{1}^{1}$ John J Leddy, ${ }^{2}$ Kevin M Guskiewicz, ${ }_{1}^{3}$ Tad Seifert, ${ }^{4}$ \\ Michael McCrea, ${ }^{5}$ Noah D Silverberg, ${ }^{6}$ Nina Feddermann-Demont, ${ }^{7,8}$ Grant L Iverson, ${ }^{9}$ \\ Alix Hayden, ${ }^{10}$ Michael Makdissi ${ }^{11,12}$
}

- Additional material is published online only. To view please visit the journal online (http://dx.doi.org/10.1136/ bjsports-2016-097475).

For numbered affiliations see end of article.

\section{Correspondence to}

Dr Kathryn J Schneider, Sport Injury Prevention Research

Centre, Faculty of Kinesiology University of Calgary, 2500 University Drive N.W. Calgary, Alberta T2N 1N4, Canada; kjschnei@ucalgary.ca

Accepted 28 February 2017 Published Online First 9 March 2017
CrossMark

To cite: Schneider KJ, Leddy JJ, Guskiewicz KM, et al. Br J Sports Med 2017;51:930-934.

\begin{abstract}
Aim or objective The objective of this systematic review was to evaluate the evidence regarding rest and active treatment/rehabilitation following sport-related concussion (SRC).

Design Systematic review.

Data sources MEDLINE (OVID), CINAHL (EbscoHost), Psyclnfo (OVID), Cochrane Central Register of Controlled Trials (OVID), SPORTDiscus (EbscoHost), EMBASE (OVID) and Proquest DissertationsandTheses Global (Proquest) were searched systematically.
\end{abstract}

Eligibility criteria for selecting studies Studies were included if they met the following criteria: (1) original research; (2) reported SRC as the diagnosis; and (3) evaluated the effect of rest or active treatment/ rehabilitation. Review articles were excluded.

Results Twenty-eight studies met the inclusion criteria (9 regarding the effects of rest and 19 evaluating active treatment). The methodological quality of the literature was limited; only five randomised controlled trials (RCTs) met the eligibility criteria. Those RCTs included rest, cervical and vestibular rehabilitation, subsymptom threshold aerobic exercise and multifaceted collaborative care.

Summary/conclusions A brief period (24-48 hours) of cognitive and physical rest is appropriate for most patients. Following this, patients should be encouraged to gradually increase activity. The exact amount and duration of rest are not yet well defined and require further investigation. The data support interventions including cervical and vestibular rehabilitation and multifaceted collaborative care. Closely monitored subsymptom threshold, submaximal exercise may be of benefit.

Systematic review registration PROSPERO 2016:CRD42016039570

\section{INTRODUCTION}

Sport-related concussion (SRC) is among the most common sport and recreation injuries. ${ }^{12}$ Most athletes recover gradually over the initial days following injury, but up to $15 \%$ have been reported to have time loss of at least 30 days. ${ }^{3}$ After a concussion, a period of cognitive and physical rest until the acute symptoms resolve followed by a progressive return to activity according to a protocol of graded exertion is recommended. ${ }^{4}$ This advice is based on expert opinion and was developed at sequential consensus meetings on concussion in sport. ${ }^{4-6}$

The symptoms and problems following concussion can vary considerably, and athletes can also experience associated injury to the cervical spine or peripheral vestibular system. Therefore, a variety of treatments may be required that address ongoing symptoms and impairments following injury. ${ }^{78}$ The purpose of this systematic review was to evaluate the evidence related to two questions: (1) What is the evidence that rest is beneficial following concussion, and is there an optimal duration of rest? (2) What is the evidence that active treatment and rehabilitation is effective for athletes who have experienced SRC?

\section{METHODS}

The initial search terms used in this systematic review, the inclusion criteria, exclusion criteria and database selection were generated and reviewed by the author group. The draft MEDLINE search strategy was then sent to an expert librarian (KAH) to ensure its completeness and accuracy. This was done according to the PRESS Guideline Statement using the CADTH Peer Review Checklist.9 The librarian revised and expanded the MEDLINE search, and then translated it for the other databases (see online supplementary tables S1 and S2). An a priori data extraction table was generated and approved by all authors.

The following electronic databases were searched: MEDLINE (OVID), CINAHL (EbscoHost), PsycInfo (OVID), Cochrane Central Register of Controlled Trials (OVID), SPORTDiscus (EbscoHost), EMBASE (OVID) and Proquest Dissertations and Theses Global (Proquest). The search was run by the expert librarian and exported for review for the author group (up to and including articles published until 17 October 2016) (see online supplementary tables S1 and S2). Duplicates were removed. References of included papers and systematic reviews were searched for additional references and authors were asked to share any additional references that met the inclusion criteria. The total yield of articles was divided into half and the title and abstract of each article was independently reviewed for inclusion by two authors for each question (four authors in total). To be considered for inclusion in this review, articles had to (1) be original research (including RCTs, quasi-experimental designs, case series, case cross-overs and case studies, cohort and case control), (2) have the focus of the study be SRC as a source of injury and (3) evaluate the effect of rest or treatment. Review articles and articles published in abstract form only were excluded. In the case of disagreement or uncertainty between the two reviewers, a third reviewer was engaged to resolve the discrepancy and where required, 
full-text manuscripts were retrieved to determine eligibility for inclusion.

Full texts were retrieved for all included articles, which were then subcategorised as per the main topics of rest and treatment. Two authors independently extracted data for each of the articles and independently evaluated the risk of bias using the Downs and Black (DB) checklist for methodological quality. ${ }^{10}$ In the case of a discrepancy, the authors discussed the difference in rating and came to a final rating. In the event that the authors were unable to come to a consensus, a third rater was engaged. We used this checklist because we anticipated a variety of study designs, and this checklist is appropriate for assessing randomised and non-randomised studies. A higher rating on the DB checklist indicates a lower risk of bias. Studies scoring below a 5 on the DB checklist were removed from the review due to a high risk of bias. Data were extracted using standardised tables and included the following: study design, participants (sample size, age, sex, sampling methods), treatment (frequency, intensity, type, timing/duration), outcome measures, key findings (point estimates with 95\% confidence intervals) and level of evidence (per Oxford Centre for Evidence Based Medicine). ${ }^{11}$

Extracted data were synthesised qualitatively. We present a qualitative synthesis of the available data regarding the effectiveness and duration of rest following SRC, and a qualitative synthesis of the available data regarding the effectiveness of treatment and rehabilitation for athletes with SRC. Meta-analyses were not performed because the intervention and outcome data were heterogeneous.

\section{RESULTS}

A total of 8224 citations were identified via the electronic database search and 2518 duplicates were removed. Four other records were identified for a total of 5710 citations. Of the four additional record identified by authors, three were not captured in the initial search as they did not mention sports or related keywords in the abstract or indexing and one did not include terms relevant to treatment or rest. A third reviewer was engaged to assess inclusion following screening of title and abstract for 35 records in which there was disagreement. Forty-one records were reviewed in full text and 29 records met the inclusion criteria (see figure 1 for PRISMA flow diagram). One study was subsequently removed from the review due to its high risk of bias $(\mathrm{DB}$ score $=2)$ resulting in a total of 28 studies (see online supplementary table S3).

A total of 3218 participants (ages 5-53) were included in this systematic review. The studies (five RCTs, nine cohort studies, two quasi-experimental studies, 12 case series) had varying scores on the DB criteria with the RCTs scoring highest-DB range: $18-25$, cohort studies-DB: 7-23, quasi-experimentalDB: $15-21$ and case series-DB: 6-16.

\section{Rest}

Nine studies examined rest following concussion (see online supplementary table S3). Six studies included both children and adults and three studies included only children. There was one eligible RCT that evaluated the effects of strict rest following SRC in adolescents and found that both groups had a $20 \%$ decrease in total energy expenditure following injury. ${ }^{12}$ The individuals who were randomised to recommendation of strict rest reported higher total symptom over 10 days and had slower symptom resolution. ${ }^{12}$ Two non-randomised treatment studies recruiting from the same specialty clinic reported benefit from a period of 1 week of prescribed cognitive and physical rest. ${ }^{13}$

${ }^{14}$ Greater activity level was associated with shorter symptom duration in one observational study ${ }^{15}$ and longer symptom duration in another. ${ }^{16}$ Two cohort studies found that self-report or parent-report of prescription of rest did not predict a longer recovery. ${ }^{17} 18$ Two retrospective studies did not find a significant association between recommended cognitive rest and time to symptom resolution. ${ }^{19}{ }^{20}$ In summary, there is conflicting evidence on the efficacy of rest following concussion on recovery of premorbid function and symptom resolution.

\section{Treatment}

Nineteen studies evaluated the effects of treatment following SRC (see online supplementary table S3). The majority (16/19 studies) reported positive effects with treatment but many were at high risk of bias (DB scores ranging from 6 to 25 with 11/19 scores below 16). Common methodological limitations included lack of a control group, lack of randomisation and lack of control for confounders. One RCT demonstrated positive effects with multimodal physiotherapy ${ }^{21}$ and two RCTs evaluated the effects of subsymptom threshold aerobic exercise training-one identifying a positive effect ${ }^{22}$ and the other found no difference with training. ${ }^{23}$ The other RCT evaluated collaborative care, including advocacy and coordination with schools regarding accommodations and motivational interviewing, cognitive-behavioural therapy (CBT) and psychopharmacological consultation. ${ }^{24}$ Other treatments that reported positive effects included a physiotherapy programme, ${ }^{25} 26$ cognitive therapies, ${ }^{27}$ medical treatments using amitriptyline, ${ }^{28}$ amantadine, ${ }^{29}$ peripheral nerve blocks (including greater occipital nerve blocks) ${ }^{30}$ and exercise interventions including submaximal aerobic training in children ${ }^{3132}$ and adults. ${ }^{33-36}$ Individuals who performed the greatest activity level were found to have worse performance in visual memory, but not other cognitive domains, in one study. ${ }^{37}$ No difference was seen in symptoms or clinical recovery when participants were exposed to a symptom-free waiting period prior to returning to play. ${ }^{38} 39$

In summary, there is moderate evidence that targeted cervical and vestibular physiotherapy is more effective than typical rest followed by graded exertion to facilitate medical clearance to return to sport. There is limited evidence that collaborative care (including CBT, psychopharmacological consultation and care management) reduces symptoms and improves health-related quality of life in youth. There is conflicting evidence that subsymptom threshold aerobic exercise decreases time to recovery; however, the majority of literature suggests a positive effect. There is no evidence to minimal evidence that medical treatments are of benefit to facilitate recovery following concussion.

\section{DISCUSSION}

Most consensus and agreement statements for managing SRC $\mathrm{SR}^{40}$ ${ }^{41}$ recommend that athletes rest until they become symptom-free. Accordingly, prescribed rest is one of the most widely used interventions in this population. ${ }^{42-45}$ At least three rationales for rest have been articulated. First, rest probably mitigates postconcussion symptoms, easing discomfort during the acute recovery period. Second, rest might promote recovery by minimising energy demands during haemodynamic and neurometabolic restoration following concussion. ${ }^{46-48}$ Third, athletes are at an increased risk for sustaining a recurrent concussion within the first 7-10 days after the initial concussion. ${ }^{38}$ A repeat concussion 


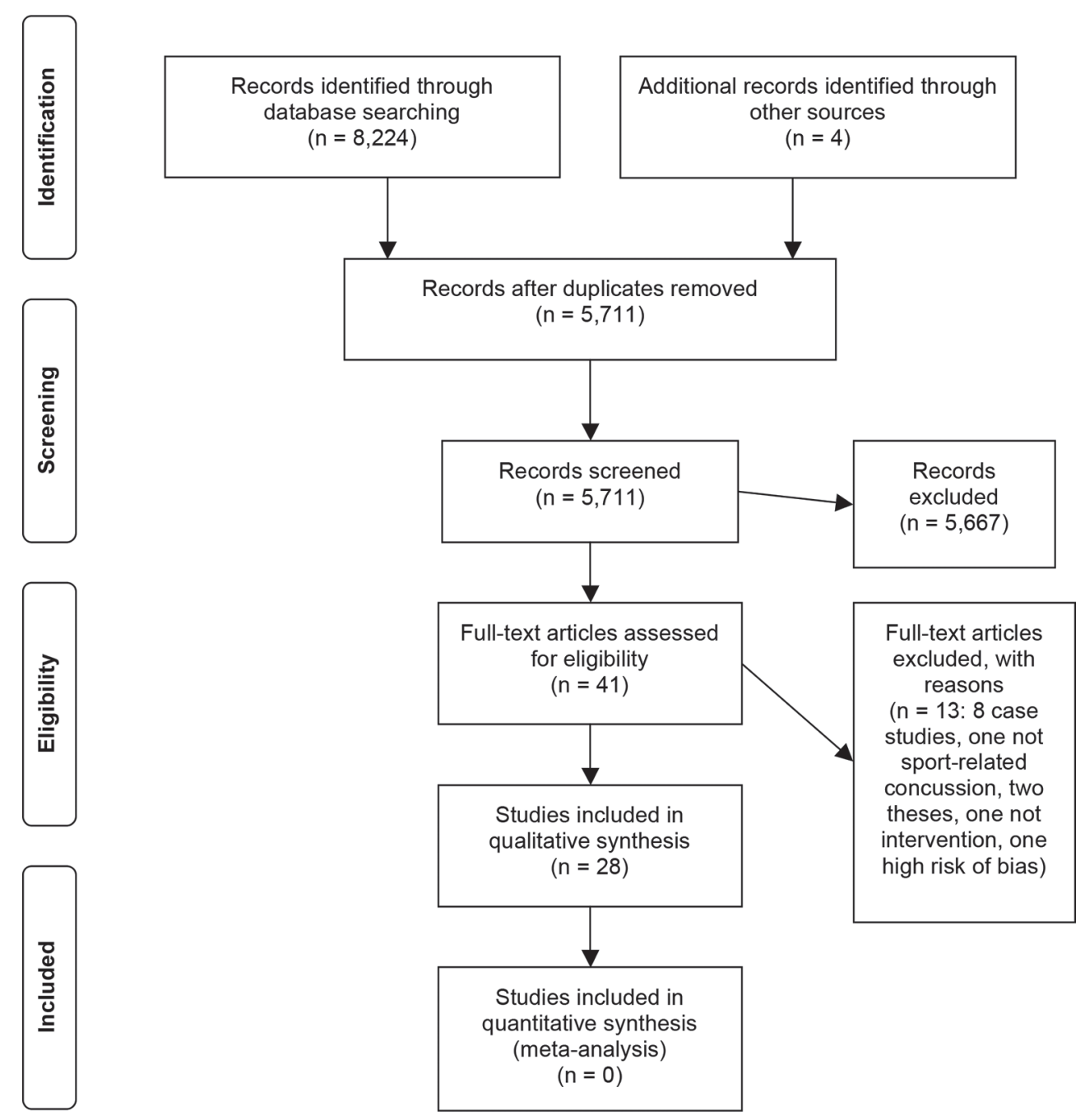

Figure 1 PRISMA flow diagram as follows: Records after duplicates removed 5710; records screened 5710 and Records excluded 5669.

during this period of recovery might have a magnified effect. ${ }^{49-51}$ Note that this last rationale for rest is for restricting sport participation and other preinjury activities with an elevated concussion risk rather than for restricting all usual activities (ie, complete rest).

There is currently insufficient evidence that prescribing complete rest facilitates recovery following SRC. Observational studies report mixed findings regarding whether physical or mental activity during the days to weeks after SRC are associated with faster or slower recovery times. ${ }^{15} 16192037$ Student athletes reported fewer symptoms and performed better on cognitive testing following a week of prescribed rest. ${ }^{13}{ }^{14}$ However, the high risk of bias in these uncontrolled and non-randomised studies leaves doubt as to whether the positive outcomes were attributable to prescribed rest versus pre-existing differences, natural recovery, expectations/placebo effects or other therapeutic aspects of the clinical encounter.

There is limited higher quality evidence from RCTs that recruited athletes and non-athletes from an emergency department. In an RCT involving adults with concussions due to mostly (90\%) non-sport causes, prescribing 6 days of complete rest (versus encouraging patients to mobilise and limit their bed rest starting on the day after injury) was associated with less dizziness in the first 4 days but bed rest was not associated with a meaningful difference in clinical outcomes at 2 weeks, 3 months or 6 months following injury. ${ }^{52}$ Adolescents with concussion who were prescribed complete rest for 5 days reported more symptoms over the 10-day observational period compared with adolescents who received usual care, typically including advice to rest for 1-2 days. ${ }^{12}$ The two groups performed similarly on balance and cognition outcomes. Of note, adherence to prescribed complete rest was modest in both these trials, which may have contributed to the small differences between the intervention groups. This also highlights the practical challenges of implementing prescribed rest. In children, self-reported physical activity within 7 days of concussion was associated with reduced risk of persistent postconcussive symptoms at 28 days following injury. $^{53}$

In summary, the best available evidence from clinical studies does not support the efficacy of prescribing complete rest for more than a few days after SRC. Both observational and experimental laboratory studies have demonstrated that bouts of intense physical or mental activity can exacerbate symptoms in concussed athletes. ${ }^{54-58}$ These exacerbations appear to be transient ${ }^{545859}$ and further research is needed to understand the underlying pathophysiology and potential impact on longterm outcomes. In the interim, athletes can be encouraged to gradually resume their non-sport activities as tolerated, avoiding heavy exertion and activities that have an elevated concussion risk until they are medically cleared. Prescribing rest for more 
than a few days should be weighed against the possible harms of prolonged activity restriction, especially in elite sports. ${ }^{60-62}$ However, shortening the rest period should not necessarily mean rapid return to sport. Timing of return to sport remains a challenge especially given the evidence for a period of vulnerability to reinjury in some athletes following concussion and should be guided by a multimodal clinical assessment.

\section{Treatment: rehabilitation}

Historically, rest followed by a protocol of graded exertion has been the treatment of choice following SRC. ${ }^{4}$ However, a more active approach to rehabilitation may be of benefit. ${ }^{72}$ 31-34 63 ${ }^{64}$ Our systematic review identified five studies that evaluated rehabilitative techniques following concussion, one of which was an RCT evaluating multimodal physiotherapy treatment (DB score 25), one RCT evaluating collaborative care (DB score 19 ) and three case series evaluating cognitive rehab (DB score 12) and multifaceted physical therapy and exercise (DB score 9 and 11). For individuals with persisting symptoms of dizziness, neck pain and headaches, a combination of cervical and vestibular rehabilitation has positive treatment effects in both youth and young adults compared with standard care (rest followed by graded exertion). ${ }^{21}$ Treatment was individualised and was based on persisting impairments in cervical and vestibular function. ${ }^{21}$

Multifaceted physiotherapy treatment regimes that include adaptation, balance, musculoskeletal, aerobic, anaerobic and sport-specific exercises might be useful. ${ }^{25}{ }^{26}$ However, poor methods (including poor description of the endpoint of return to full activity) limit the conclusions that can be drawn.

There is a large body of evidence demonstrating positive treatment effects with vestibular rehabilitation for individuals with vestibular disorders. ${ }^{65-67}$ Vestibular rehabilitation is safe and effective for peripheral vestibular disorders including unilateral peripheral vestibular hypofunction and benign paroxysmal positional vertigo ${ }^{65-68}$ Additionally, studies evaluating the effects of vestibular rehabilitation have demonstrated positive symptomatic and functional improvements in patients who have suffered mild traumatic brain injuries (mTBI) of all types, including blast injuries. ${ }^{69-71}$ Thus, vestibular rehabilitation may be appropriate for individuals with persistent vestibular findings following SRC.

A combination of specific exercises and manual therapy that focus on function is effective for improving pain and function in individuals with cervical spine pain and cervicogenic headaches. ${ }^{72}$ Thus, inclusion of exercise and manual therapy in the case of ongoing cervical spine pain and cervicogenic headaches following concussion may be of benefit.

For individuals with mTBI, there is some evidence to support the use of an educational pamphlet ${ }^{737}$ and $\mathrm{CBT}^{75-78} \mathrm{~A}$ recent RCT that employed a multifaceted collaborative treatment protocol including motivational interviewing, school accommodations, CBT and psychopharmacological therapy (where indicated) identified a positive treatment effect on postconcussive symptoms and health-related quality of life. ${ }^{24}$ Further research to determine the efficacy of rehabilitative strategies evaluating other areas that may be affected by SRC such as cognition, mood, psychological disorders, sleep disturbance and others, in isolation as well as in combination, is required.

\section{Treatment: exercise}

In articles included in our systematic review, concussed male and female athletes with persistent symptoms lasting between 1 and 12 months were typically introduced to subsymptom submaximal (light) aerobic exercise multiple times per week. Overall, oursystematic review supports the notion that controlled exercise performed at an intensity and duration that does not exacerbate symptoms is likely safe and beneficial for adult athletes with persistent symptoms following concussion. Athletes in the submaximal exercise groups typically reported fewer symptoms, recovered to baseline on cognitive and balance scores faster, and in one study ${ }^{34}$ demonstrated more efficient activation patterns on functional Magnetic Resonance Imaging (fMRI) relative to matched controls. One RCT in adolescents demonstrated a positive treatment effect with subsymptom exacerbation aerobic training on symptom ratings; however, poorer adherence to exercise was reported in the aerobic training group compared with the stretching group. ${ }^{79}$ The other RCT demonstrated no difference between groups in time to recovery. ${ }^{80}$

Studies involving children and adolescent athletes experiencing symptoms for at least 4 weeks following concussion showed similar improvements after completion of a progressive rehabilitation programme. ${ }^{3132}$ All young athletes returned to a normal lifestyle and full sport participation. While more robust RCTs are needed, these findings suggest that closely monitored active rehabilitation programmes involving controlled subsymptom threshold, submaximal exercise for adults and adolescents with persistent symptoms after concussion may be of benefit.

\section{Treatment: medical}

Three studies on medical treatment of SRC were identified. All studies were retrospective case series with moderate risk of bias scores. Two of the studies described the treatment of post-traumatic headaches (PTH) with either interventional or traditional pharmacological measures. There is limited evidence to support various medical management strategies for SRC, including the treatment of PTH. There are no available data from RCTs evaluating the therapeutic efficacy of medical interventions specific to PTH (in athletes or otherwise), although a small number of case reports and cohort studies have recently been published. The data from studies suggesting that certain pharmacotherapeutics may be effective treatment strategies in the context of PTH have not shown that medical intervention hastens recovery following SRC. ${ }^{28} 8182$

\section{Treatment: other (symptom-free waiting period)}

The results of the two studies evaluating the effects of a symptom-free waiting period suggest that outcomes, such as risk of reinjury, may be more closely associated with an initial period of vulnerability to reinjury than with apparent symptom resolution. Therefore, these findings highlight the importance of injury recognition and prudent return to play decision making by clinicians during the first 10 days after SRC.

Two RCTs in our review included interventions that involved more than one type of treatment and demonstrated positive effects. ${ }^{2463}$ Because concussion is an injury with heterogeneous symptom and functional presentations of patients, multifaceted rehabilitation protocols may be of benefit to facilitate recovery. Further research in this area is needed.

\section{Limitations}

Most of the studies included in this review were of low methodological quality and biased by systematic errors. Our review may be subject to a publication bias. This review may be subject to a language bias as we only included articles published in the English language. We only included studies that evaluated rest or treatment for SRC. There may be other treatments of potential 
benefit that have been evaluated in this population but were not identified by our inclusion criteria.

\section{Suggestions for future research}

The current literature contains few high-quality studies and many of the current studies included in this review have a high risk of bias. Future studies should evaluate the optimal timing, mode, duration, intensity and frequency of treatment during the postacute time period following concussion, ideally through RCTs, where feasible. To facilitate research and improve the clinical care of PTH, use of the International Classification of Headache Disorders (ICHD) 3 beta standardised criteria is preferable due to its precise classification methods. Further research evaluating rest and active treatments should be performed using high-quality designs that account for potential confounding factors and effect modifiers.

\section{CONCLUSION}

After a brief period (24-48 hours) of complete rest, patients can be encouraged to become gradually and progressively more active while staying below their cognitive and physical symptom exacerbation thresholds. Athletes should avoid heavy exertion and activities with an elevated risk of head injury while they are recovering. The exact amount and duration of rest is not yet well defined.

A variety of treatments may be required for ongoing symptoms and impairments following concussion. Interventions including cervical and vestibular rehabilitation (for individuals with persisting dizziness, cervical spine pain and headaches) and closely monitored active rehabilitation programmes involving controlled subsymptom threshold, submaximal exercise could be considered. Specific treatment recommendations can be directed based on clinical examination findings and symptoms.

\section{Author affiliations}

${ }^{1}$ Faculty of Kinesiology, University of Calgary, Calgary, Alberta, Canada

${ }^{2}$ Department of Orthopaedics, SUNY Buffalo, Buffalo, New York, USA

\section{What is already known?}

- An initial period of cognitive and physical rest following concussion is recommended-followed by a progression of gradually increasing activity.

- The literature evaluating the effects of treatment following concussion is limited.

\section{What are the new findings?}

- The exact amount and duration of rest is not yet well defined.

- After a brief period (24-48 hours) of complete rest, patients can be encouraged to become gradually and progressively more active while staying below their cognitive and physical symptom exacerbation thresholds.

- Research supports interventions including cervical and vestibular rehabilitation (for individuals with persisting dizziness, cervical spine painand headaches). Closely monitored active rehabilitation programmes involving subsymptom threshold and submaximal exercise are safe and may facilitate recovery. Multifaceted collaborative treatments (including cognitive-behavioural therapy, school accommodations and pharmacotherapy) may also be of benefit.
${ }^{3}$ Sports Medicine Research laboratory, University of North Carolina at Chapel Hill, Chapel Hill, North Carolina, USA

${ }^{4}$ Norton Healthcare, Louisville, Kentucky, USA

${ }^{5}$ Department of Neurosurgery, Medical College of Wisconsin, Milwaukee, Wisconsin, USA

${ }^{6}$ Department of Medicine, University of British Columbia, Vancouver, British Columbia, Canada

${ }^{7}$ Department of Neurology, University Hospital Zurich, Zurich, Switzerland

${ }^{8}$ Schulthess Clinic, Zurich, Switzerland

${ }^{9}$ Physical Medicine and Rehabilitation, Harvard Medical School; and Red Sox Foundation and Massachusetts General Hospital Home Base Program, Boston, Massachusetts, USA

${ }^{10}$ Libraries and Cultural Resources, University of Calgary, Calgary, Alberta, Canada ${ }^{11}$ Melbourne Brain Centre, Florey Institute of Neuroscience and Mental Health Austin Campus, Heidelberg, Victoria, Australia

${ }^{12}$ Olympic Park Sports Medicine Centre, Melbourne, Australia

Competing interests KS has received speaking honoraria for presentations at scientific meetings. She is a physiotherapy consultant at Evidence Sport and Spinal Therapy in Calgary, Alberta, Canada and for athletic teams. KG is the Founding Director of the Matthew Gfeller Sport-Related TBI Research Center at the University of North Carolina, USA. He is a member of the National Collegiate Athletic Association's Concussion Committee and the US Soccer Federation (unpaid consultant for both). KG at times receives honoraria and reimbursement of expenses to speak at professional meetings. TS is a member of the Speakers Bureau, Avanir Pharmaceuticals. MMC receives research funding from the National Collegiate Athletic Association (NCAA). NS reports a research salary grant from the Vancouver Coastal Health Research Institute, grants and consultant fees from WorkSafeBC, consultant fees from the National Hockey League, receiving salary from the Vancouver Coastal Health Authority and Home Base, a Red Sox Foundation and Massachusetts General Hospital programme, outside the submitted work. $\mathrm{GI}$ has been reimbursed by the government, professional scientific bodies and commercial organisations for discussing or presenting research relating to MTBI and sport-related concussion at meetings, scientific conferences and symposiums. He has a clinical practice in forensic neuropsychology involving individuals who have sustained mild TBIs (including athletes). He receives support from the Harvard Integrated Program to Protect and Improve the Health of National Football League Players Association Members. He acknowledges unrestricted philanthropic support from the Mooney-Reed Charitable Foundation and ImPACT Applications, Inc. MM is a consultant Sport and Exercise Medicine physician at Olympic Park Sports Medicine Centre and team physician for the Hawthorn football club (Australian Football League, AFL). He receives research funding from the Australian Football League (AFL) and non-financial support from CogState Pty Ltd. He has attended meetings organised by the IOC, National Football League (USA), National Rugby League (Australia) and FIFA (Switzerland); however has not received any payment, research funding, or other monies from these groups other than for travel costs. He is an honorary member of concussion working/advisory groups for AFL, Australian Rugby Union and World Rugby.

Provenance and peer review Not commissioned; externally peer reviewed.

(c) Article author(s) (or their employer(s) unless otherwise stated in the text of the article) 2017. All rights reserved. No commercial use is permitted unless otherwise expressly granted.

\section{REFERENCES}

1 Emery CA, Kang J, Shrier I, et al. Risk of injury associated with body checking among youth ice hockey players. Jama 2010;303:2265-72.

2 Black AM, Sergio LE, Macpherson AK. The epidemiology of concussions: number and nature of concussions and time to recovery among female and male Canadian varsity Athletes 2008 to 2011. Clin J Sport Med 2017;27:52-6.

3 Kerr ZY, Zuckerman SL, Wasserman EB, et al. Concussion symptoms and return to play time in youth, high school, and college American football Athletes. JAMA Pediatr 2016;170:647.

4 McCrory P, Meeuwisse WH, Aubry M, et al. Consensus statement on concussion in sport: the 4th international conference on concussion in sport held in Zurich, November 2012. Br J Sports Med 2013;47:250-8.

5 McCrory P, Johnston K, Meeuwisse W, et al. Summary and agreement statement of the 2nd international conference on concussion in sport, Prague 2004. Br J Sports Med 2005;39:i78-i86.

6 McCrory P, Meeuwisse W, Johnston K, et al. Consensus statement on concussion in sport 3rd international conference on concussion in sport held in Zurich, November 2008. Clin J Sport Med 2009;19:185-200.

7 Schneider KJ. Sport-Related concussion: optimizing treatment through EvidenceInformed practice. J Orthop Sports Phys Ther 2016;46:613-6.

8 Marshall S, Bayley M, McCullagh S, et al. Clinical practice guidelines for mild traumatic brain injury and persistent symptoms. Can Fam Physician 2012;58:257e128-6740. 
9 McGowan J, Sampson M, Salzwedel DM, et al. PRESS peer review of electronic search strategies: 2015 guideline Statement. J Clin Epidemiol 2016;75:40-6.

10 Downs SH, Black N. The feasibility of creating a checklist for the assessment of the methodological quality both of randomised and non-randomised studies of health care interventions. J Epidemiol Community Health 1998;52:377-84.

11 Oxford Level of Evidence Working Group, 2011. Oxford Centre for Evidence-Based Medicine 2011 Levels of Evidence

12 Thomas DG, Apps JN, Hoffmann RG, et al. Benefits of strict rest after acute concussion: a randomized controlled trial. Pediatrics 2015;135:213-23.

13 Moser RS, Schatz P, Glenn M, et al. Examining prescribed rest as treatment for adolescents who are slow to recover from concussion. Brain Inj 2015;29:58-63.

14 Moser RS, Schatz P. A case for mental and physical rest in youth sports concussion: it's never too late. Front Neurol 2012;3:171.

15 Howell DR, Mannix RC, Quinn B, et al. Physical activity level and symptom duration are not associated after concussion. Am J Sports Med 2016;44:1040-6.

16 Brown NJ, Mannix RC, O'Brien MJ, et al. Effect of cognitive activity level on duration of post-concussion symptoms. Pediatrics 2014;133:e299-e304.

17 Corwin DJ, Zonfrillo MR, Master CL, et al. Characteristics of prolonged concussion recovery in a pediatric subspecialty referral population. J Pediatr 2014;165:1207-15.

18 Eisenberg MA, Andrea J, Meehan W, et al. Time interval between concussions and symptom duration. Pediatrics 2013;132:8-17.

19 Gibson S, Nigrovic LE, O'Brien M, et al. The effect of recommending cognitive rest on recovery from sport-related concussion. Brain Inj 2013;27:839-42.

20 Moor HM, Eisenhauer RC, Killian KD, et al. The relationship between adherence behaviors and recovery time in adolescents after a sports-related concussion: an observational study. Int J Sports Phys Ther 2015;10:225-33.

21 Schneider K, Meeuwisse W, Nettel-Aguirre A, et al. Cervico-vestibular physiotherapy in the treatment of individuals with persistent symptoms following sport-related concussion: a randomized controlled trial. Brain Injury 2014;28(5-6):796.

22 Kurowski BG, Hugentobler J, Quatman-Yates C, et al. Aerobic exercise for adolescents with prolonged symptoms after mild traumatic brain injury: an exploratory randomized clinical trial. J Head Trauma Rehabil 2016.

23 Maerlender A, Rieman W, Lichtenstein J, et al. Programmed physical exertion in recovery from Sports-Related concussion: a randomized pilot study. Dev Neuropsychol 2015;40:273-8.

24 McCarty CA, Zatzick D, Stein E, et al; Seattle Sports Concussion Research Collaborative. Collaborative care for adolescents with persistent postconcussive symptoms: a randomized trial. Pediatrics 2016;138:e20160459.

25 Hugentobler JA, Vegh M, Janiszewski B, et al. Physical therapy intervention strategies for patients with prolonged mild traumatic brain injury symptoms: a case series. Int J Sports Phys Ther 2015;10:676-89.

26 Grabowski P, Wilson J, Walker A, et al. Multimodal impairment-based physical therapy for the treatment of patients with post-concussion syndrome: a retrospective analysis on safety and feasibility. Phys Ther Sport 2017;23:22-30.

27 Sohlberg MM, Ledbetter AK. Management of persistent cognitive symptoms after Sport-Related concussion. Am J Speech Lang Pathol 2016;25:138-49.

28 Bramley H, Heverley S, Lewis MM, et al. Demographics and treatment of adolescent posttraumatic headache in a regional concussion clinic. Pediatr Neurol 2015;52:493-8.

29 Reddy CC, Collins M, Lovell M, et al. Efficacy of amantadine treatment on symptoms and neurocognitive performance among adolescents following sports-related concussion. J Head Trauma Rehabil 2013;28:260-5

30 Dubrovsky AS, Friedman D, Kocilowicz H. Pediatric post-traumatic headaches and peripheral nerve blocks of the scalp: a case series and patient satisfaction survey. Headache 2014;54:878-87.

31 Gagnon I, Galli C, Friedman D, et al. Active rehabilitation for children who are slow to recover following sport-related concussion. Brain Inj 2009;23:956-64.

32 Gagnon I, Grilli L, Friedman D, et al. A pilot study of active rehabilitation for adolescents who are slow to recover from sport-related concussion. Scand I Med SC Sports 2016;26:299-306.

33 Leddy JJ, Kozlowski K, Donnelly JP, et al. A preliminary study of subsymptom threshold exercise training for refractory post-concussion syndrome. Clin J Sport Med 2010;20:21-7

34 Leddy JJ, Cox JL, Baker JG, et al. Exercise treatment for postconcussion syndrome: a pilot study of changes in functional magnetic resonance imaging activation, physiology, and symptoms. J Head Trauma Rehabil 2013;28:241-9.

35 Clausen M, Pendergast DR, Willer B, et al. Cerebral blood flow during treadmill exercise is a marker of physiological postconcussion syndrome in female Athletes. J Head Trauma Rehabil 2016;31:215-24.

36 Cordingley D, Girardin R, Reimer K, et al. Graded aerobic treadmill testing in pediatric sports-related concussion: safety, clinical use, and patient outcomes. J Neurosurg Pediatr 2016;25:693-702.

37 Majerske CW, Mihalik JP, Ren D, et al. Concussion in sports: postconcussive activity levels, symptoms, and neurocognitive performance. J Ath/ Train 2008;43:265-74.

38 McCrea M, Guskiewicz K, Randolph C, et al. Effects of a symptom-free waiting period on clinical outcome and risk of reinjury after sport-related concussion. Neurosurgery 2009;65:876-83.
39 Pfaller AY, Nelson LD, Apps JN, et al. Frequency and outcomes of a Symptom-Free waiting period after Sport-Related concussion. Am J Sports Med 2016;44:2941-6.

40 Broglio SP, Cantu RC, Gioia GA, et al; National Athletic Trainer's Association. National athletic trainers' Association position statement: management of sport concussion. $J$ Athl Train 2014:49:245-65.

41 DeMatteo C, Stazyk K, Singh SK, et al; Ontario Neurotrauma Foundation. Development of a conservative protocol to return children and youth to activity following concussive injury. Clin Pediatr 2015;54:152-63.

42 Lebrun CM, Mrazik M, Prasad AS, et al. Sport concussion knowledge base, clinical practises and needs for continuing medical education: a survey of family physicians and cross-border comparison. Br J Sports Med 2013;47:54-9.

43 Arbogast KB, McGinley AD, Master CL, et al. Cognitive rest and school-based recommendations following pediatric concussion: the need for primary care support tools. Clin Pediatr 2013;52:397-402.

44 Stoller J, Carson JD, Garel A, et al. Do family physicians, emergency department physicians, and pediatricians give consistent sport-related concussion management advice? Can Fam Physician 2014;60:548-52.

45 Zemek R, Eady K, Moreau K, et al. Canadian pediatric emergency physician knowledge of concussion diagnosis and initial management. Cjem 2015:17:115-22

46 Giza CC, Hovda DA, Da H. The neurometabolic cascade of concussion. J Athl Train 2001:36:228-35

47 Barkhoudarian G, Hovda DA, Giza CC. The molecular pathophysiology of concussive brain injury - an update. Phys Med Rehabil Clin N Am 2016;27:373-93.

48 Wells EM, Goodkin HP, Griesbach GS. Challenges in determining the role of rest and exercise in the management of mild traumatic brain injury. J Child Neurol 2016:31:86-92.

49 Vagnozzi R, Tavazzi B, Signoretti S, et al. Temporal window of metabolic brain vulnerability to concussions: mitochondrial-related impairment--part I. Neurosurgery 2007;61:379-89.

50 Longhi L, Saatman KE, Fujimoto $S$, et al. Temporal window of vulnerability to repetitive experimental concussive brain injury. Neurosurgery 2005;56:364-74.

51 Laurer HL, Bareyre FM, Lee VM, et al. Mild head injury increasing the brain's vulnerability to a second concussive impact. J Neurosurg 2001;95:859-70.

52 de Kruijk JR, Leffers $P$, Meerhoff $S$, et al. Effectiveness of bed rest after mild traumatic brain injury: a randomised trial of no versus six days of bed rest. J Neurol Neurosurg Psychiatry 2002;73:167-72.

53 Grool AM, Aglipay M, Momoli F, et al; Pediatric Emergency Research Canada (PERC) Concussion Team. Association between early participation in physical activity following acute concussion and persistent postconcussive symptoms in children and adolescents. Jama 2016:316:2504-14

54 Balasundaram AP, Sullivan JS, Schneiders AG, et al. Symptom response following acute bouts of exercise in concussed and non-concussed individuals - a systematic narrative review. Phys Ther Sport 2013;14:253-8.

55 Leddy JJ, Baker JG, Kozlowski K, et al. Reliability of a graded exercise test for assessing recovery from concussion. Clin J Sport Med 2011;21:89-94.

56 Dematteo C, Volterman KA, Breithaupt PG, et al. Exertion testing in youth with mild traumatic brain injury/Concussion. Med Sci Sports Exerc 2015;47:2283-90.

57 McGrath N, Dinn WM, Collins MW, et al. Post-exertion neurocognitive test failure among student-athletes following concussion. Brain Inj 2013;27:103-13.

58 Silverberg ND, Iverson GL, McCrea M, et al. Activity-Related symptom exacerbations after pediatric concussion. JAMA Pediatr 2016;170:946-53.

59 Brooks BL, Low TA, Daya $\mathrm{H}$, et al. Test or rest? computerized cognitive testing in the emergency department after pediatric mild traumatic brain injury does not delay symptom recovery. J Neurotrauma 2016;33:2091-6.

60 DiFazio M, Silverberg ND, Kirkwood MW, et al. Prolonged activity restriction after concussion: are we worsening outcomes? Clin Pediatr 2016:55:443-51.

61 Silverberg ND, Iverson GL. Is rest after concussion "the best medicine?": recommendations for activity resumption following concussion in athletes, civilians, and military service members. J Head Trauma Rehabil 2013;28:250-9.

62 Craton N, Leslie 0 . Is rest the best intervention for concussion? lessons learned from the whiplash model. Curr Sports Med Rep 2014;13:201-4.

63 Schneider KJ, Meeuwisse WH, Nettel-Aguirre A, et al. Cervicovestibular physiotherapy in the treatment of individuals with persistent symptoms following sport related concussion: a randomised controlled trial. British Journal of Sports Medicine 2014;48:1294-8

64 Peters M, Price J. The peters/price (see to play) vision concussion protocol: diagnosis and treatment? Optometry \& Visual Performance 2015;3:126-38.

65 Hilton M, Pinder D. The epley manoeuvre for benign paroxysmal positional Vertigo--a systematic review. Clin Otolaryngol Allied Sci 2002;27:440-5.

66 Hilton M, Pinder D. The epley (canalith repositioning) manoeuvre for benign paroxysmal positional Vertigo. Cochrane Database Syst Rev 2004;2:CD003162.

67 Hillier S, Hollohan V. Vestibular rehabilitation for unilateral peripheral vestibular dysfunction. Cochrane Database Syst Rev 2007;17:CD005397.

68 Shepard NT, Telian SA, Smith-Wheelock M, et al. Vestibular and balance rehabilitation therapy. Ann Otol Rhinol Laryngol 1993;102:198-205. 
69 Alsalaheen BA, Mucha A, Morris LO, et al. Vestibular rehabilitation for dizziness and balance disorders after concussion. J Neurol Phys Ther 2010;34:87-93.

70 Gottshall K. Vestibular rehabilitation after mild traumatic brain injury with vestibular pathology. NeuroRehabilitation 2011;29:167-71.

71 Gottshall K, Gray N, Drake Al. A unique collaboration of female medical providers within the united states armed forces: rehabilitation of a marine with postconcussive vestibulopathy. Work 2005;24:381-6.

72 Gross A, Kay TM, Goldsmith C, et al; Cervical Overview Group. Exercises for mechanical neck disorders. Cochrane Database Syst Rev 2015;28:CD004250.

73 Ponsford J, Willmott C, Rothwell A, et al. Impact of early intervention on outcome after mild traumatic brain injury in children. Pediatrics 2001;108:1297-303.

74 Ponsford J, et al. Impact of early intervention on outcome following mild head injury in adults. Journal of Neurology, Neurosurgery \& Psychiatry 2002;73:330-2.

75 Ponsford J, Lee NK, Wong D, et al. Efficacy of motivational interviewing and cognitive behavioral therapy for anxiety and depression symptoms following traumatic brain injury. Psychol Med 2016;46:1079-90.

76 Tiersky LA, Anselmi V, Johnston MV, et al. A trial of neuropsychologic rehabilitation in mild-spectrum traumatic brain injury. Arch Phys Med Rehabil 2005;86:1565-74.
77 Snell DL, Surgenor L, Hay-Smith EJ, et al. A systematic review of psychological treatments for mild traumatic brain injury: an update on the evidence. I Clin Exp Neuropsychol 2009;31:20-38.

78 Nygren-de Boussard C, Holm LW, Cancelliere C, et al. Nonsurgical interventions after mild traumatic brain injury: a systematic review. results of the international collaboration on mild traumatic brain injury prognosis. Arch Phys Med Rehabil 2014;95:S257-S264.

79 Kurowski BG, Hugentobler J, Quatman-Yates C, et al. Aerobic exercise for adolescents with prolonged symptoms after mild traumatic brain injury: an exploratory randomized clinical trial. J Head Trauma Rehabil 2016.

80 Maerlender A, Rieman W, Lichtenstein J, et al. Programmed physical exertion in recovery from Sports-Related concussion: a randomized pilot study. Dev Neuropsychol 2015;40:273-8.

81 Gladstone J. From psychoneurosis to ICHD-2: an overview of the state of the art in post-traumatic headache. Headache 2009;49:1097-111.

82 Kuczynski A, Crawford S, Bodell L, et al. Characteristics of post-traumatic headaches in children following mild traumatic brain injury and their response to treatment: a prospective cohort. Dev Med Child Neurol 2013;55:636-41. 